\title{
Quantification et caractérisation de la matière organique de sols alluviaux au cours de l'évolution de la végétation
}

\author{
M Fierz 1, JM Gobat 1, C Guenat 2 \\ 1 Laboratoire d'écologie végétale, Institut de botanique, Chantemerle 22, 2000 Neuchâtel; \\ 2 Laboratoire de pédologie, GR-IATE, École polytechnique fédérale, 1015 Lausanne, Suisse
}

(Reçu le 1er août 1994; accepté le 18 mars 1995)

\begin{abstract}
Résumé - Les auteurs identifient les formes et déterminent les quantités de matière organique de l'humus de 2 séries de sols alluviaux - Fluviosols (les sols dont le nom commence par une majuscule sont désignés selon le Référentiel pédologique. Principaux sols d'Europe (AFES, 1992) -, l'une sur alluvions à dominante calcaire et l'autre sur alluvions à dominante d'orthogneiss, dans le but d'établir si les caractères physico-chimiques de cette matière organique correspondent au degré d'évolution traduit par la morphologie du sol, d'une part, et par la végétation actuelle, d'autre part. Ils réalisent un premier fractionnement par tamisage à l'eau, destiné à séparer et à quantifier la matière végétale figurée et la matière végétale la plus humifiée. Sur cette dernière, ils procèdent à des extractions chimiques sélectives. Les résultats démontrent que la quantité (stock en carbone et en azote) de matière organique est un meilleur indicateur que la qualité, qui ne varie pratiquement pas en milieu calcaire et que très lentement en milieu acide. La vitesse d'évolution de l'humus de ces sols par rapport à celle de la végétation présente en moins d'un siècle une inertie marquée qui laisse entrevoir un décalage dans l'adéquation sol-végétation.
\end{abstract}

relation sol-végétation / humus / qualité et quantité de matière organique / sol alluvial

Summary - Organic matter quantification and characterization of alluvial soils during vegetation evolution. In order to determine if the physical and chemical criterias of organic matter are in phase with the evolutive state expressed both by the soil morphology and by the present vegetation, forms of organic matter are identified and then quantified in the humus of 2 different series of alluvial soils (Fluviosols). One series is situated on mainly calcareous alluvia and the other on alluvia principally constituted of orthogneiss (figs 1 and 2; table I). A first fractionation, using the method of water sieving, is undertaken to separate and to estimate the particulate plant material from the best humified plant material. The latter is then selectively chemically extracted (fig 3; table III). Results show that the amount (stock in carbon and nitrogen) of organic matter is a better indicator (table II) than its quality, which virtually 
does not change in a calcareous environment and only slowly in acid conditions (tables $I V$ and $V$ ). As compared with the vegetation dynamic, the slow humus evolution in these soils seems to reveal in less than 100 years a shift in the soil-vegetation relationship.

alluvial soils / humus / organic matter forms and quantity / soil-vegetation relationship

\section{INTRODUCTION}

Depuis quelques années, les pays européens se préoccupent de la sauvegarde des milieux humides et plus particulièrement, sous l'impulsion du Conseil de l'Europe [recommandation $n^{\circ} \mathrm{R}(82) 12,1981$ ], de celle des zones alluviales dont l'importance biologique n'est plus à démontrer. En Suisse, l' «inventaire des zones alluviales d'importance nationale" (Kuhn et Amiet, 1988) ainsi que la cartographie de la végétation (Gallandat et al, 1993) qui en résultent ont mis en évidence l'appauvrissement de ces écosystèmes. Les processus d'évolution en conditions anthropisées ou naturelles, en particulier ceux qui intéressent les sols, doivent être élucidés afin de proposer une gestion adéquate telle que la prévoit l'«ordonnance sur la protection des zones alluviales d'importance nationale" (Conseil fédéral, 1992), entrée en vigueur le 15 novembre 1992.

L'étude de l'évolution des sols fait depuis longtemps appel aux caractéristiques minérales de la couverture pédologique (Duchaufour, 1983). Les caractéristiques de la matière organique, quant à elles, n'ont pu être exploitées que suite à la mise au point récente de protocoles et techniques analytiques (par ex, Stevenson, 1982). Ainsi, la connaissance des caractéristiques des composés organiques permet d'aborder l'étude de sols très peu différenciés au point de vue pédogénétique comme les sols alluviaux (Fluviosols), les sols peu évolués minéraux (Régosols) ou humifères (Organosols) et les sols tourbeux (Histosols) (Schnitzer, 1978).
En effet, la matière organique, réagissant plus vite que les minéraux au changement des conditions du milieu, permet de détecter des étapes extrêmement précoces d'évolution et de suivre presque en temps réel les stades pionniers de formation des sols. Elle autorise également des comparaisons avec la dynamique de la végétation, dont la qualité de la litière influence les caractéristiques de la matière organique.

Dans un premier temps, nous cherchons à mettre en évidence les caractères physico-chimiques de la matière organique les plus aptes à rendre compte d'une maturation des sols. Lors d'une seconde étape, nous tentons d'interpréter les résultats obtenus en terme de degré d'évolution des sols, parallèlement à celui de la végétation.

\section{MATÉRIEL ET MÉTHODES}

\section{Sols et végétation}

Notre étude porte sur 2 séquences de sols, l'une caractérisée par une pédogenèse sur alluvions à dominante calcaire (bassin de la Sarine) et l'autre par une pédogenèse sur alluvions à dominante d'orthogneiss (bassin de la Maggia). Chaque séquence comporte 3 profils choisis à partir d'une série évolutive de végétation. Le temps écoulé entre les différents stades est déduit de la comparaison entre l'âge du peuplement arborescent actuel (mesures dendrométriques) et l'étude diachronique de la colonisation par la végétation (photos aériennes). Le manque de documents photographiques concernant les stades les plus évolués rend les estimations plus délicates.

La première séquence (fig 1) comprend, du stade pionnier au stade forestier, des Fluviosols 
typiques carbonatés, à texture limono-sableuse, qui se distinguent les uns des autres par leur épaisseur, notamment celle de leur humus, et leur taux de carbonates. Le stade initial porte une saulaie blanche (âge du peuplement : 13 ans ; station colonisée depuis 13 ans environ). Les stades intermédiaire et ultime sont respectivement caractérisés par une frênaie (âge du peuplement : 38 ans; station colonisée depuis plus de 60 ans) et une hêtraie (âge du peuplement : 148 ans ; temps de colonisation supérieur à 113 ans). Ces sols sont formés sous climat tempéré froid et humide $\left(P_{\text {moy }}=1,200 \mathrm{~mm} ; T_{\text {moy }}=7^{\circ} \mathrm{C}\right)$, à l'étage montagnard inférieur (alt $\mathrm{t}_{\text {moy }}=730 \mathrm{~m}$ ), et se situent dans les préalpes fribourgeoises (Suisse). La seconde séquence (fig 2), qui intègre un Fluviosol typique polyphasé sous aulnaie blanche (âge du peuplement : 25 ans ; colonisation de la station depuis 31 ans), un Fluviosol typique polyphasé peu évolué sous frênaie (âge du peuplement : 25 ans; colonisation de la station depuis 31 ans) et un Fluviosol sous chênaie à charmes (âge du peuplement : 35 ans ; temps de colonisation : 61 ans). Ces sols, sableux, sont régis par un climat tempéré chaud à caractère insubrien (orages violents et soudains en été) $\left(P_{\text {moy }}=1,600 \mathrm{~mm} ; T_{\text {moy }}=9-10^{\circ} \mathrm{C}\right)$, à l'étage col- linéen (alt $\mathrm{t}_{\text {moy }}=350 \mathrm{~m}$ ), et se trouvent dans les Alpes du Sud (Tessin, Suisse).

II faut noter que les stades pionniers sont, du fait de leur position topographique, soumis à des rajeunissements partiels imperceptibles sur les photos aériennes. Les données analytiques essentielles des humus de ces 2 séquences sont présentées dans le tableau l.

\section{Méthodes de fractionnement}

Nous avons procédé à un fractionnement physique [tamisage à l'eau, Bruckert et Kilbertus (1980)] pour isoler une fraction supposée humifiée (fraction fine, $\leq 100 \mu \mathrm{m}$ ). Dans un second temps, des extractions chimiques $[\mathrm{NaOH} 0,1 \mathrm{~N} ; \mathrm{pH}=$ 12 ; Bruckert et Kilbertus (1980)] ont été réalisées sur le sol total et sur la fraction fine. Le rapport entre le carbone $(C)$ [ou l'azote $(N)$ ] de la fraction fine pondérée et le $\mathrm{C}$ (ou N) du sol total, exprimé en \%, représente le taux d'humification (Andreux, 1981).

Suite à des modifications suggérées par Andreux (communication orale, 1991), le protocole mentionné figure 3 a été appliqué.

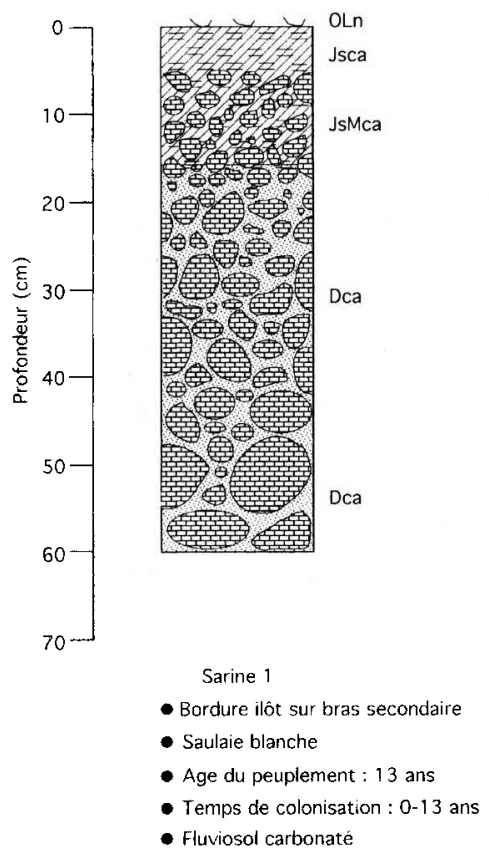

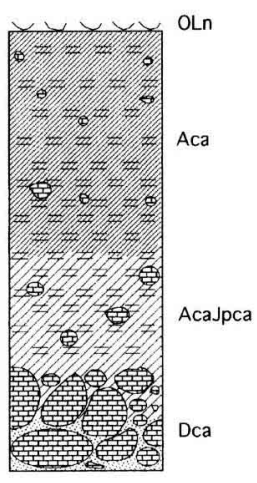

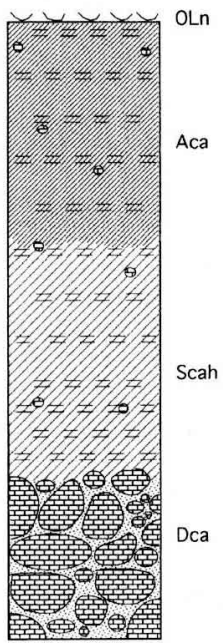

Sarine 2

- Terrasse inondable

- Frènaie

- Age du peuplement : 38 ans

- Temps de colonisation : $>60$ ans

- Fluviosol carbonaté
Sarine 3

- Ancienne terrasse

- Hétraie

- Age du peuplement : 148 ans

- Temps de colonisation : > 113 ans

- Fluviosol carbonaté à tend. brunifiée

Fig 1. Les 3 profils types de la Sarine (Préalpes fribourgeoises). 


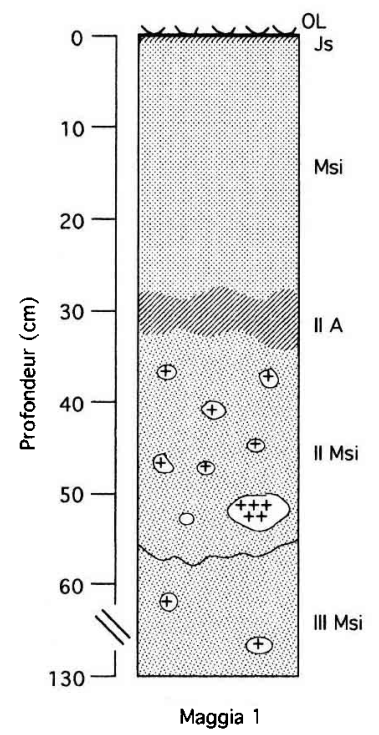

- llot sur bras secondaire

- Aulnaie blanche

- Age du peuplement : 25 ans

- Temps de colonisation : 31 ans

- Fluviosol polyphasé

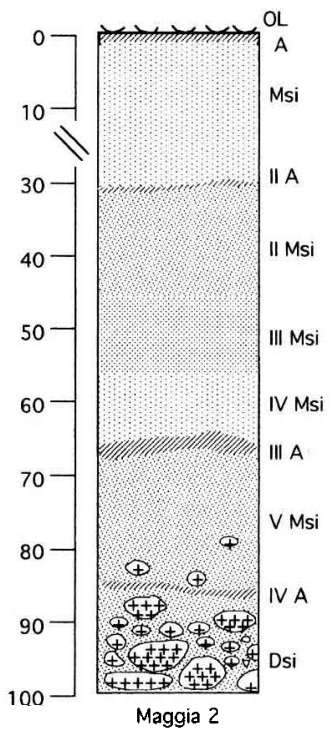

- Terrasse inondable

- Frênaie

- Age du peuplement : 25 ans

- Temps de colonisation : 31 ans

- Fluviosol polyphasé peu évolué

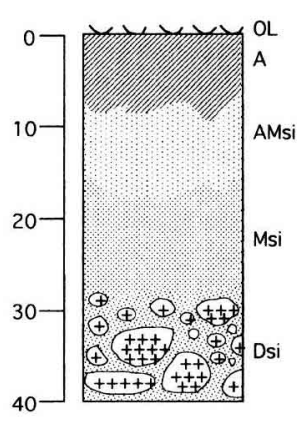

Maggia 3

- Ancienne terrasse

- Chênaie à charme

- Age du peuplement : 35 ans

- Temps de colonisation : 61 ans

- Fluviosol

Fig 2. Les 3 profils types de la Maggia (Alpes du Sud, Tessin).

\section{Analyse des fractions}

Les dosages du carbone et de l'azote des fractions granulométriques, $2000-100 \mu \mathrm{m}$ et 100 $0 \mu \mathrm{m}$, ont été respectivement réalisés sur l'appareil Casumat 8-Adge Wösthoff et sur un photomètre Technicon après minéralisation des échantillons selon la méthode Kjeldahl. Les extraits sous forme liquide ont été dosés sur l'appareil TOC Astro pour le carbone et selon le procédé évoqué ci-dessus pour l'azote.

\section{RÉSULTATS}

\section{Concentration et stock de C et $\mathbf{N}$ organiques du sol total}

Si les concentrations en carbone et en azote organiques de l'humus des échantillons de sol total (tableaux I et III) augmentent parallèlement au degré d'évolution supposé du sol mais de manière discrète dans chaque série, il en va tout autrement lorsque I'on exprime ces mêmes résultats en termes volumiques, calculés en tenant compte de la concentration de carbone ou d'azote, de l'épaisseur et de la densité de l'horizon humifère (tableau II). On observe alors, aussi bien pour l'azote que pour le carbone, un accroissement significatif qui, du stade pionnier (1) au stade forestier (3), avoisine un facteur dix.

\section{Fractionnement physique}

Les résultats obtenus (tableau III) indiquent que l'importance pondérale de la fraction 100-0 $\mu \mathrm{m}$ augmente pour chaque série du 


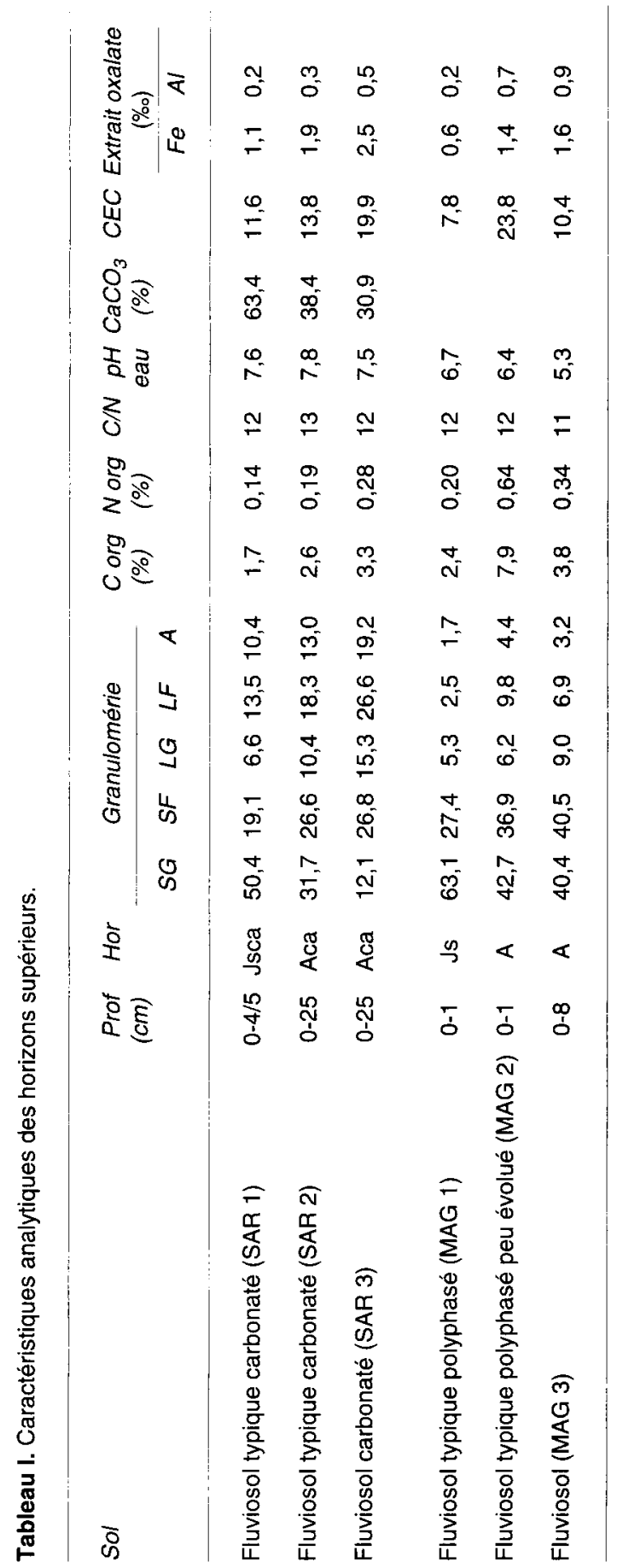




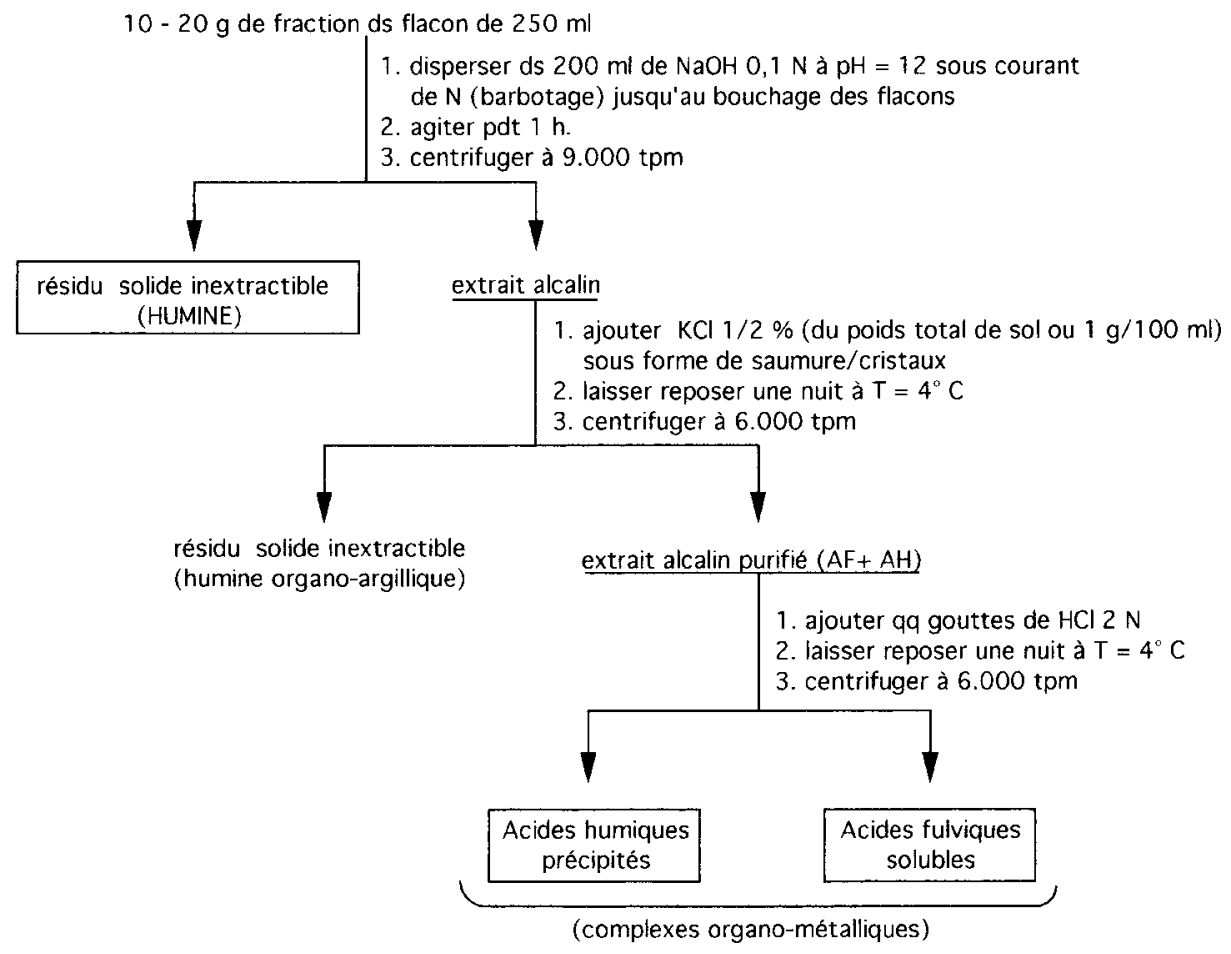

Fig 3. Protocole de fractionnement chimique.

stade 1 à 3 . Cette fraction est majoritaire dans le cas de la série carbonatée (58 à $86 \%$ ), plus faible dans la série sur orthogneiss (32 à 44\%). Les concentrations en carbone et en azote organiques de la fraction fine suivent la même évolution excepté pour MAG 2. Les taux d'humification du carbone et de l'azote augmentent dans les 2 séries de manière quasi similaire. Les valeurs obtenues sont beaucoup plus élevées pour les sols calcaires mais demeurent cependant inférieures à celles citées pour les sols eutrophes et mésotrophes (Andreux et Corréa, 1981).

Les rapports $\mathrm{C} / \mathrm{N}$ du sol total montrent peu de variations même lorsque la comparaison intègre les 2 types de sols. À une exception près, les fractions grossières se distinguent des fractions fines par des valeurs toujours plus élevées; ces dernières présentent d'ailleurs des rapports $\mathrm{C} / \mathrm{N}$ proches des taux réalisés par les échantillons de sol total, comme déjà observé par Andreux et Correa (1981).

\section{Fractionnement chimique (tableaux IV et V)}

Ce procédé a permis d'isoler 3 types de matière organique: les acides fulviques $(A F)$, les acides humiques (AH) et l'humine (H) dans lesquels ont été dosés le carbone et l'azote organiques

Dans tous les humus, le carbone organique se trouve essentiellement sous forme 
Tableau II. Teneurs volumiques en $\mathrm{C}$ et $\mathrm{N}$ organiques du sol total.

Sol

Fluviosol typique carbonaté (SAR 1)

Fluviosol typique carbonate (SAR 2)

Fluviosol typique carbonaté (SAR 3)

Fluviosol typique polyphasé (MAG 1)

Fluviosol typique polyphasé peu évolué (MAG 2)

Fluviosol (MAG 3)

d'humine aussi bien dans la fraction fine que dans le sol total. Le taux d'extraction

$$
\frac{C_{A F}+C_{A H}}{C_{\text {sol tot ou fraction }}} \times 100 \text {, }
$$

faible, est compris entre 3 et $5 \%$ pour la série "Sarine» ; il augmente dans la série «Maggia» et atteint pour le sol total et la fraction fine respectivement 16 et $24 \%$ dans le cas de MAG 3. Quant aux rapports $\mathrm{AF} / \mathrm{AH}$, ils sont voisins de 1 ; seule la fraction fine du sol le plus évolué de la série «Maggia» accuse une baisse importante $(0,6)$. Le taux d'extraction de l'azote suit à peu près le même cours que celui du carbone.

Les rapports $\mathrm{C} / \mathrm{N}$ des $\mathrm{AF}$ sont faibles pour l'ensemble des humus, de l'ordre de 5 à 8 ; ils augmentent pour les $\mathrm{AH}$ et les humines avec un maximum dans le cas des $\mathrm{AH}$ de la série sur gneiss (24).

\section{DISCUSSION}

\section{Intérêt de l'approche quantitative}

À la lecture de nos résultats, il apparaît que les teneurs volumiques en carbone et azote organiques sont les meilleurs indicateurs

\begin{tabular}{|c|c|c|}
\hline Épaisseur horizon $(\mathrm{cm})$ & $C_{\text {org }}(t / h a)$ & $N_{\text {org }}(t / h a)$ \\
\hline 5 & 8,6 & 0,7 \\
\hline 25 & 64,3 & 4,8 \\
\hline 25 & 83,3 & 7,0 \\
\hline 1 & 2,4 & 0,2 \\
\hline 1 & 7,9 & 0,6 \\
\hline 8 & 30,5 & 2,7 \\
\hline
\end{tabular}

du degré d'évolution du sol. Ce rôle primordial de révélateur d'étapes précoces d'évolution est confirmé dans une étude similaire effectuée sur des sols artificiels, composés de débris de roche calcaire brute mélangés à du compost (Gobat et Strehler, 1993). Dans ces mélanges élaborés en fonction d'un taux de carbone organique croissant, les auteurs mettent en évidence la formation d'agrégats organo-minéraux après quelques années. Cette structuration ne se traduit guère par les critères chimiques habituels de la matière organique (taux d'extraction, rapport $\mathrm{AF} / \mathrm{AH}$, rapport $H / C)$, mais s'exprime plutôt par sa répartition dans les différentes classes granulométriques, quelle que soit la proportion rochecompost du mélange. Systématiquement, la quantité de carbone organique diminue après quelques années dans la classe $2000-100 \mu \mathrm{m}$ (déchets figurés du compost) pour augmenter au profit de la classe 50-5 $\mu \mathrm{m}$ qui, selon Bruckert (1994), correspond à la limite de séparation des complexes organo-minéraux pour des échantillons faiblement humifiés.

Dans la présente étude, on assiste à un processus identique de concentration du carbone et de l'azote dans la fraction fine $(\leq 100 \mu \mathrm{m})$. Cette tendance s'allie à une différenciation morphologique du profil, accompagnée d'une altération avec décarbonatation ou acidification, d'une libération d'argile 


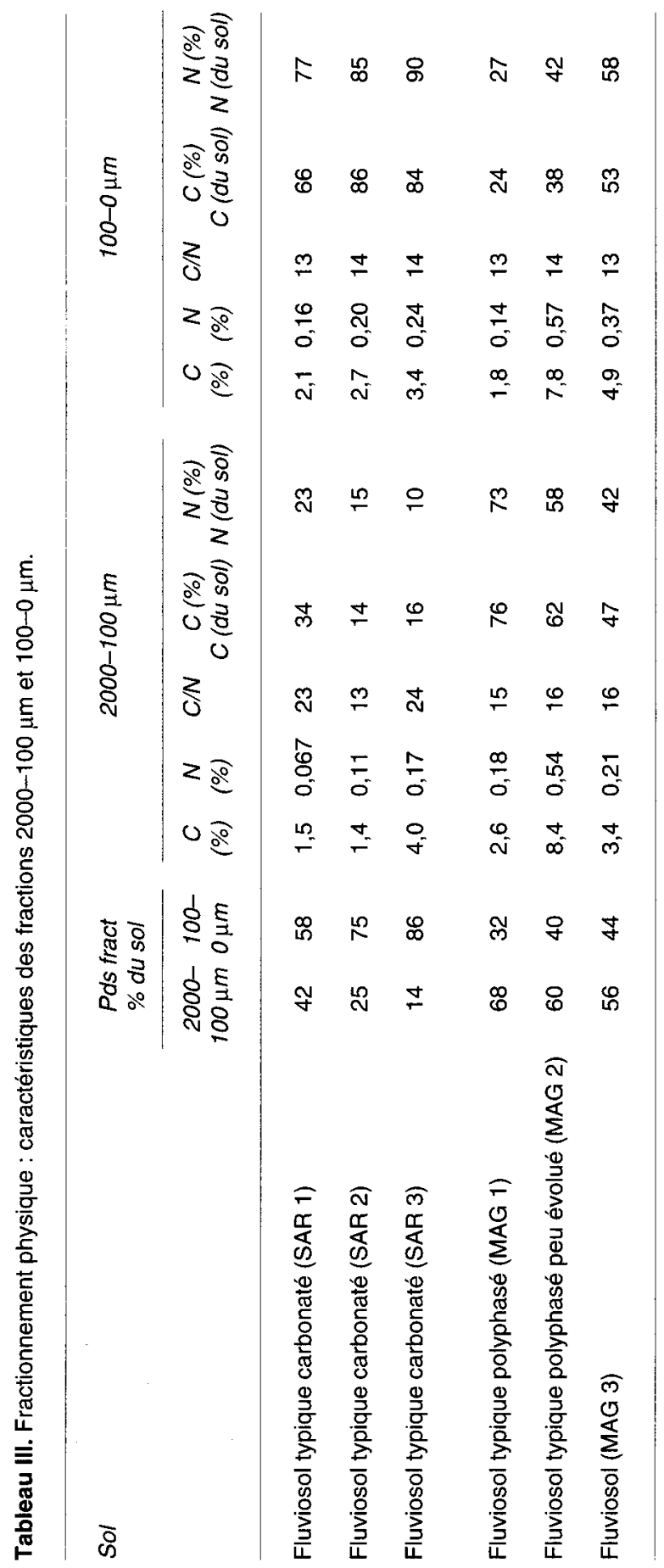




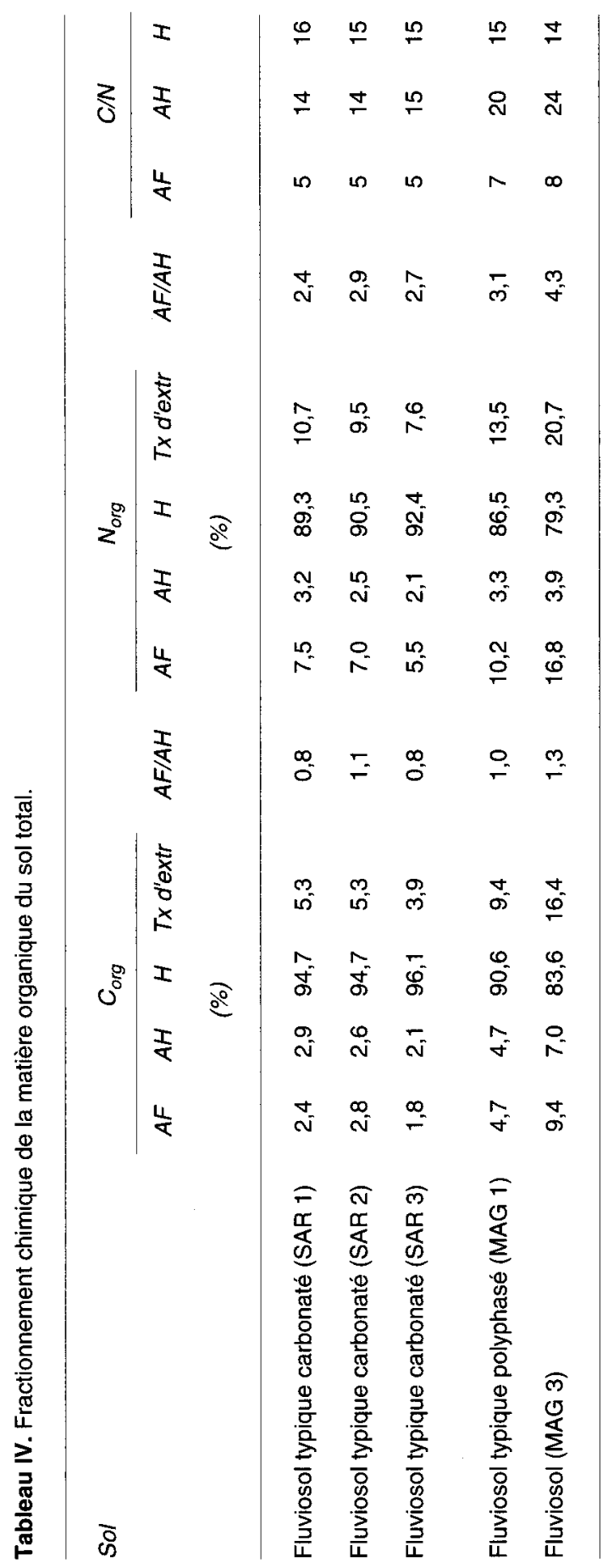




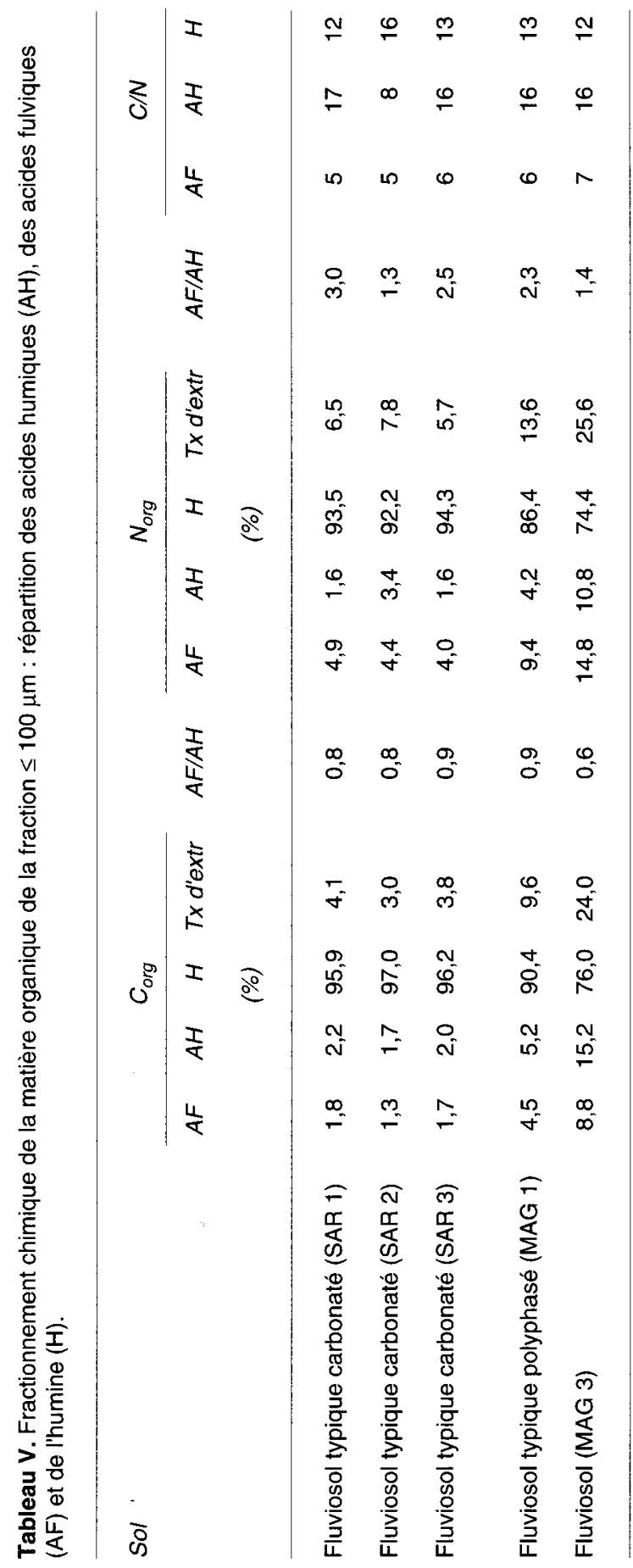


et de fer amorphe associée à une structure de mieux en mieux exprimée. Ce phénomène est particulièrement marqué dans le cas de la série des sols carbonatés, limonosableux, tandis qu'il reste discret dans le cas de la série sur gneiss où des teneurs faibles en argile et en fer sont liées à des temps d'évolution limités (moins d'un siècle). Ainsi, l'augmentation pondérale de la fraction fine et la stabilisation du carbone et de l'azote organiques dans cette même fraction seraient un indice de maturation de l'humus dont l'efficacité dépend du temps et du substrat. Ce concept est partagé par Carbiener (communication orale) et Pautou (1984) qui considèrent l'apparition du complexe argilo-humique comme un bon indicateur du degré d'évolution des sols alluviaux. À l'échelle temporelle, Walker et Coventry (1987) soulignent l'importance du carbone et de l'azote organiques en tant que signes de changement. Nos travaux confirment ces faits pour des séquences évoluant en quelques décennies, voire en une centaine d'années.

\section{Limites de l'approche qualitative}

On pouvait s'attendre à ce que la qualité de la matière organique, exprimée par des indicateurs tels que le taux d'extraction, le taux d'acides fulviques ou humiques ou encore le taux d'humine, montre une progression qui suit celle de la différenciation morphologique du sol. Les résultats montrent en fait peu de modifications au plan chimique, l'humine demeurant toujours prépondérante aussi bien dans le sol total que dans la fraction fine. De ce fait, il s'agit vraisemblablement d'humine héritée. Cette hypothèse devrait être étayée par d'autres approches. La seule tendance évolutive décelable est celle qui s'exprime dans le cas du sol morphologiquement le plus évolué sur gneiss (MAG 3) au travers d'une augmentation du taux d'extraction et, dans la fraction fine uni- quement, d'une diminution du rapport AF/AH.

Cette quasi-absence d'évolution chimique pourrait être la conséquence :

- d'un blocage précoce de l'évolution de la matière organique sur calcaire (effet de ciment des carbonates) ;

- d'une certaine lenteur de l'évolution sur substrat gneissique, due à une activité biologique réduite, résultant de stress hydriques (pluviométrie irrégulière, mise en réserve limitée) et d'une capacité d'échange cationique faible liée à l'absence d'argile ;

- d'une minéralisation et d'une humification lentes en raison du type de litière, souvent constituée de débris ligneux (branchettes d'arbres et de buissons), qui ne semble pas être compensée par une litière de feuilles pourtant nettement améliorante (Salix, Alnus, Fraxinus) ; une partie de ces débris pourraient être hérités de stations de résineux situées en amont. Cette dépendance étroite entre l'amont et l'aval, décrite, à propos de la matière organique notamment, par Décamps et Naiman (1989), est une caractéristique propre de la dynamique alluviale.

Toutefois, on ne peut pas contester la valeur des informations résultant des analyses chimiques de la matière organique. II faudrait effectuer des recherches plus poussées en établissant par exemple des spectres d'absorption infrarouge ou RMN révélant les fonctions chimiques des acides organiques (Andreux, 1994 ; Orlov, 1985 ; Schnitzer, 1978 ; Stevenson, 1982). De même, les hydrates de carbone peuvent aussi s'avérer très utiles pour la classification des sols (Lowe, 1978).

Les séquences évolutives proposées sur la base des caractéristiques morphologiques du sol et de séries dynamiques de la végétation ont donc été confirmées par les critères analytiques des humus. On note que la teneur volumique de carbone et d'azote ainsi que la concentration de ces éléments dans les fractions fines vont de pair avec 
l'altération de la phase minérale. En conclusion, si la formation d'agrégats requiert une certaine qualité de matière organique, celleci n'a toutefois pas pu être mise en évidence dans ce type de sols très peu évolués.

\section{Évolution comparée du sol et de la végétation}

La comparaison du stade d'évolution perçu au travers de descripteurs pédologiques et de descripteurs de la végétation, notamment structurel, floristique et phytosociologique révèle un décalage. Ainsi, la hêtraie et la chênaie, situées actuellement sur des terrasses hors d'atteinte des crues (coloniées respectivement depuis plus de 148 et 61 ans), sont déjà proches d'un état d'équilibre à caractère non alluvial (climax climatique) alors qu'elles se développent sur des sols morphologiquement peu différenciés et dont la qualité de l'humus varie peu. Les prémices d'une maturation chimique plus poussée n'ont pu être décelées que dans le cas des sols sur substrat gneissique. Si le temps de réponse aux modifications du milieu est souvent plus lent pour le sol que pour la végétation, il est étonnant de constater ici qu'il l'est tout autant pour la matière organique bien que cette dernière soit directement influencée par les retombées de litière de la végétation actuelle.

\section{CONCLUSION}

La typologie des sols fait généralement appel à toute une série de caractères descriptifs et analytiques et aboutit fréquemment au diagnostic du degré d'évolution. Si les critères communément admis ne posent aucun problème dans des sols déjà relativement mûrs, aux horizons A développés, il n'en va pas de même dans le cas de sols très «jeunes», comme les sols alluviaux ou les sols artificiels. Les descripteurs habituels perdent de leur pertinence et l'on est tenu de se limiter à certains aspects de la matière organique, voire à un élément chimique ; on cite par exemple l'azote, qui diminue rapidement après un changement d'affectation du sol (Campbell, 1978). Les 2 études dont il est question ici, l'une sur des sols alluviaux et l'autre sur des sols artificiels, ont montré que les mesures quantitatives de la matière organique (stock de carbone et d'azote) étaient les plus riches de renseignements. Un véritable rôle diagnostique du stock organique du sol est mis en évidence, aidant à la compréhension des stades "très initiaux" de la pédogenèse. II s'agit là, nous semble$t$-il, d'un outil efficace et relativement simple de détection des stades précoces de l'évolution de sols jeunes, surtout lorsque l'on désire les comparer entre eux.

N'oublions pas que nous exposons ici des processus pédogénétiques qui s'amorcent en quelques années seulement, laps de temps souvent considéré par les pédologues comme «imperceptibles» en regard du temps d'évolution général des sols. L'utilisation de la matière organique comme indicateur, notamment sa quantité, nous permet d'aborder ces termes initiaux de la typologie et de l'évolution des sols.

\section{REMERCIEMENTS}

Les auteurs adressent leurs remerciements à JC Védy, directeur du laboratoire de pédologie de l'École polytechnique fédérale de Lausanne, qui a mis à notre disposition l'infrastructure et le matériel indispensables à l'élaboration de ce travail, ainsi qu'à $\mathrm{F}$ Bureau, doctorant, qui nous a apporté une aide précieuse.

\section{RÉFÉRENCES}

AFES (1992) Référentiel pédologique. Principaux sols d'Europe. INRA, Paris

Andreux $F$, Correa A (1981) Caractères généraux de la matière organique de sols eutrophes et mésotrophes affectés ou non par des migrations particulaires. In : 
Migrations organo-minérales dans les sols tempérés, CR Coll Int CNRS 303, Nancy, 329-339

Andreux F, Munier-Lamy C (1994) Genèse et propriétés des molécules humiques. IV. Technologie des substances humiques. In : Pédologie 2. Constituants et propriétés du sol (M Bonneau, B Souchier, eds), Masson, Paris, 125-127

Bruckert S, Kilbertus G (1980) Fractionnement et analyse des complexes organo-minéraux de sols bruns et de chernozems. Plant Soil 57, 271-295

Bruckert S (1994) Analyse des complexes organo-minéraux des sols. III. Méthodes de fractionnement des matières organiques et des complexes organo-minéraux. In : Pédologie 2. Constituants et propriétés du sol (M Bonneau, B Souchier, eds), Masson, Paris. 279-288

Campbell CA (1978) Soil organic carbon, nitrogen and fertility. Effects of management on soil organic matter. In : Soll Organic Matter. Developments in Soil Science 8 (M Schnitzer, SU Khan, eds), Elsevier. Amsterdam, 185-201

Conseil fédéral. Ordonnance sur la protection des zones alluviales d'importance nationale (Ordonnance sur les zones alluviales) du 28 octobre 1992, RO 1992. RS 451.31

Décamps H, Naiman RJ (1989) L'écologie des fleuves. La Recherche 208, 310-319

Duchaufour P (1983) Pédologie 1. Pédogenèse et classification. Masson, Paris

Gallandat JD, Gobat JM, Roulier C (1993) Cartographie des zones alluviales d'importance nationale. Cahier de l'environnement, nature et paysage, OFEFP, Berne

Gobat JM, Strehler C (1993) Analyse de la matière organique de sols artificiels. Rapport université de Neuchâtel et bureau Biol-Conseils SA, Neuchâtel (inédit)

Kuhn N, Amiet R (1988) Inventaire des zones alluviales d'importance nationale. Département fédéral de l'intérieur, Berne

Lowe LE (1978) Carbohydrates in soil. Carbohydrate distribution in soil. In : Soil Organic Matter. Developments in Soil Science 8 (M Schnitzer, SU Khan, eds), Elsevier, Amsterdam, 67-74

Orlov DS (1985) Humus acids of soils. Balkema, Rotterdam

Pautou G (1984) L'organisation des forêts alluviales dans l'axe rhodanien entre Genève et Lyon ; comparaison avec d'autres systèmes fluviaux. Doc Cart Evol XXVII, 43-64

Schnitzer M (1978) Humic substances: chemistry and reactions. In: Soll Organic Matter. Developments in Soil Science 8 (M Schnitzer, SU Khan, eds), Elsevier, Amsterdam, 1-64

Stevenson FJ (1982) Humus Chemistry. Genesis, Composition, Reactions. J Wiley and Sons, New York, NY, USA

Walker PH, Coventry RJ (1987) Soil profile development in some alluvial deposits of eastern New South Wales. Australian Jour Soil Res 14, 305-317. In: Alluvial Soils (J Gerrard, ed), Van Nostrand Reinhold Company, New York, NY, USA, 192-204 